\title{
Invited Article:
}

\section{Human natural autoantibodies in the treatment of neurologic disease}

Moses Rodriguez, MD

Arthur E. Warrington, $\mathrm{PhD}$

Larry R. Pease, PhD

Address correspondence and reprint requests to Dr. Moses Rodriguez, Department of Neurology, Mayo Medical School, Guggenheim 442B, 200 First Street, SW, Rochester, MN 55905

rodriguez.moses@mayo.edu

\section{ABSTRACT}

Naturally occurring autoantibodies are molecules that are part of the normal immunoglobulin repertoire. This review focuses on three distinct groups of human monoclonal antibodies (mAb). These are human natural autoantibodies that, when injected into an animal model of human disease, stimulate remyelination in CNS demyelinating diseases, protect neurons and extend neuronal processes in CNS axonal disorders, and activate immune dendritic cells to produce cytotoxic $T$ cells to clear metastatic tumors. Natural autoantibodies react to self antigens and are of relatively low affinity. They are derived from germline immunoglobulin genes and are usually polyreactive. Our experiments demonstrated CNS entry by autoradiography of labeled $\mathrm{mAb}$ and by MRI. Remyelinating $\mathrm{mAb}$ rHIgM22 clusters beta-integrin and mouse mAb $\mathrm{O} 4$ recognizes sulfatide. Neuronal outgrowth mAbs sHIgM42 and sHIgM12 appear to target carbohydrates on the surface of neurons. The mAb sHIgM12 (B7-DC-Xab) also is promising as therapeutic against metastatic tumors. It functions by binding and crosslinking the antigen B7-DC on dendritic cells, inducing tumor-specific cytotoxic T cells. All these mAbs activate a transient increase in intracellular calcium, signal via NF $\kappa b$, and prevent apoptosis. The mAbs engage downstream signaling events that induce the primary function of the cell (that is, remyelination for oligodendrocytes, axonal preservation and neurite extension for neurons, or antigen presentation for dendritic cells). Natural human auto mAbs are a potentially important therapeutic technique in combating a wide spectrum of disease processes. Neurology ${ }^{\circledR} 2009 ; 72: 1269-1276$

\section{GLOSSARY}

Ig = immunoglobulin; $\mathbf{m A b}=$ monoclonal antibodies; $\mathbf{M S}=$ multiple sclerosis .

Naturally occurring autoantibodies are a subgroup of monoclonal antibodies $(\mathrm{mAb})$ that are part of our human immunoglobulin repertoire. ${ }^{1}$ They are naturally made primarily from our own immunoglobulin genes usually without major somatic mutations. Dr. Statis Avrameas ${ }^{2}$ and others at the Pasteur Institute described their existence as early as the 1970s. They proposed that the molecules play a natural physiologic function (relating to normal cell processes), either to stimulate cell processes or to remove cellular debris. Some natural autoantibodies have been shown to recognize cytokines and growth factors. ${ }^{1}$

We discuss three distinct groups of mAbs discovered in our laboratories. These are all human natural autoantibodies that, when injected into an animal model of human disease, play an important role in remyelinating lesions in CNS demyelinating diseases, ${ }^{3}$ protecting neurons and extending neuronal processes in CNS axonal disorders, ${ }^{4}$ or binding to immune dendritic cells to stimulate the generation of cytotoxic $\mathrm{T}$ cells to clear metastatic tumors. $^{5}$

From the Departments of Immunology (M.R., L.R.P.) and Neurology (M.R., A.E.W.), Mayo Clinic College of Medicine, Rochester, MN. Supported by grants from the NIH (R01 NS 24180, R01 NS 32129, P01 NS 38468, R01 CA104996, R01 CA096859), the National Multiple Sclerosis Society (RG 317 2-B-8, CA 1011 A8-3), the Multiple Sclerosis Society of Canada, the Applebaum Foundation, the Hilton Foundation, the Peterson Foundation, and Acorda Therapeutics, Inc. (Hawthorne, NY).

Disclosure: Patents for promotion of remyelination and stimulation of dendritic cells are issued and are owned by Mayo Foundation. Therefore, the authors have a potential financial conflict of interest. 
The recombinant monoclonal proteins derived from the DNA sequences of these mAbs have been generated and have shown similar therapeutic functions as the natural $\mathrm{mAb}{ }^{6}{ }^{6}$ Two of these recombinant natural $\mathrm{mAbs}$ are being generated for clinical trials. The $\mathrm{mAb}$ that stimulates dendritic cells to generate cytotoxic $\mathrm{T}$ cells is already in Phase I clinical trials at the Mayo Clinic in patients with metastatic melanoma. The recombinant form of the $\mathrm{mAb}$ that promotes remyelination is in the late phases of animal toxicology before phase I human trial. We hope this new class of therapeutic molecules will have efficacy in human disease.

CHARACTERISTICS OF NATURAL AUTOANTIBODIES Naturally occurring autoantibodies react to self antigens, whereas conventional antibodies react to exogenous antigens, and compared to conventional antibodies, natural autoantibodies are of relatively low affinity. They are derived from our germline immunoglobulin (Ig) genes but can also contain somatic mutations. They are frequently polyreactive. They are more frequently IgMs rather than IgGs and are usually physiologic (relating to normal cell processes), unlike conventional antibodies, which are blocking or pathologic. ${ }^{7}$

This review focuses on human and mouse naturally occurring autoantibodies discovered in our laboratories. Our discussion of remyelinating mAbs focuses on recombinant human 22 and 46 (rHIgM22 and $\mathrm{rHIgM} 46)^{3}$ and mouse mAb O4. ${ }^{8}$ The antigen recognized by $\mathrm{mAb} \mathrm{O} 4$ is sulfatide. The neuronal outgrowth mAbs, specifically sHIgM42 and sHIgM12, appear to be directed against carbohydrates on gangliosides on neurons. The IgM mAb that has yielded promising results against metastatic tumors is B7-DC-XAb. It is a membrane microdomain cross-linking $\mathrm{mAb}$ that binds to $\mathrm{B} 7-\mathrm{DC}$ on dendritic cells to induce the growth and activation of cytotoxic $\mathrm{T}$ cells. Because these mAbs are polyreactive, possibly more than one antigen needs to be recognized for the biologic function.

ROLE OF AUTOANTIBODIES IN THE PROMOTION OF CNS REMYELINATION Animal model. Our discovery of the function of natural autoantibodies for CNS remyelination was accidental. At the point of discovery, we were trying to induce more demyelination in an animal model of demyelinating disease (Theiler virus infection) to test the hypothesis of virus-induced autoimmunity. However, when we immunized animals with myelin months after Theiler virus infection, as opposed to extensive demyeli- nation, ${ }^{9}$ we observed extensive remyelination. ${ }^{10}$ As a result, we performed a classic passive transfer experiment in which we transferred antisera or purified immunoglobulins from uninfected animals immunized with myelin antigens into animals that had extensive chronic demyelination following Theiler infection. ${ }^{11,12}$ Of interest, the animals receiving hyperimmune sera or immunoglobulin directed against myelin showed extensive remyelination in contrast to animals that received the control antisera.

We developed a therapeutic strategy to test the extent of remyelination. We infected animals with Theiler virus and then waited 6 or 9 months for severe, extensive demyelination to develop. We then treated the animals for 5 weeks with our mAbs and then evaluated them for spinal cord remyelination. As has been shown in other models, remyelination is a very fast phenomenon. Within 5 weeks, remyelination was almost complete. ${ }^{4}$

The first remyelination-promoting $\mathrm{mAb}$ that we identified was spinal cord homogenate 94.03 ( $\mathrm{SCH}$ 94.03). ${ }^{13}$ This was a mouse $\mathrm{mAb}$ generated in the laboratory in an animal that was immunized with spinal cord homogenate. This IgM mAb, when injected peritoneally, induced almost complete remyelination in approximately $30 \%$ of the lesions in Theiler virus-induced demyelination. In contrast, the control antibody showed remyelination in less than $5 \%$ of lesions. This $\mathrm{mAb}$ also decreased relapses in experimental autoimmune encephalomyelitis. ${ }^{14} \mathrm{Sim}$ ilar to other natural mAbs, it was polyreactive. ${ }^{15}$

Discovering natural human autoantibodies. Over the next 5 years, we identified eight different mouse mAbs that promoted remyelination. ${ }^{8}$ Of note, each one of those mAbs bound to the surface of oligodendrocytes. ${ }^{16}$ They all had relatively conserved germline sequences. ${ }^{17,18}$ Based on this observation, we asked whether natural autoantibodies could be present in the human population. We sought patients with disease processes that cause them to make their own mAbs, specifically patients with multiple myeloma, Waldenstrom syndrome, and monoclonal gammopathy of unknown significance. We had over 125,000 samples available to us archived at the Mayo Clinic by Dr. Robert Kyle from patients with monoclonal gammopathies. We screened for binding to sliced live cerebellum and, specifically, to oligodendrocytes in culture. Two mAbs that we screened, sHIgM22 and sHIgM46, demonstrated extensive remyelination $^{3}$ (figure 1) in both Theiler virus and in lysolecithin-induced demyelination. ${ }^{19}$

Having observed that the remyelination-inducing $\mathrm{mAbs}$ bind to the surface of rat and mouse oligodendrocytes, ${ }^{16}$ we next needed to determine whether the hu- 

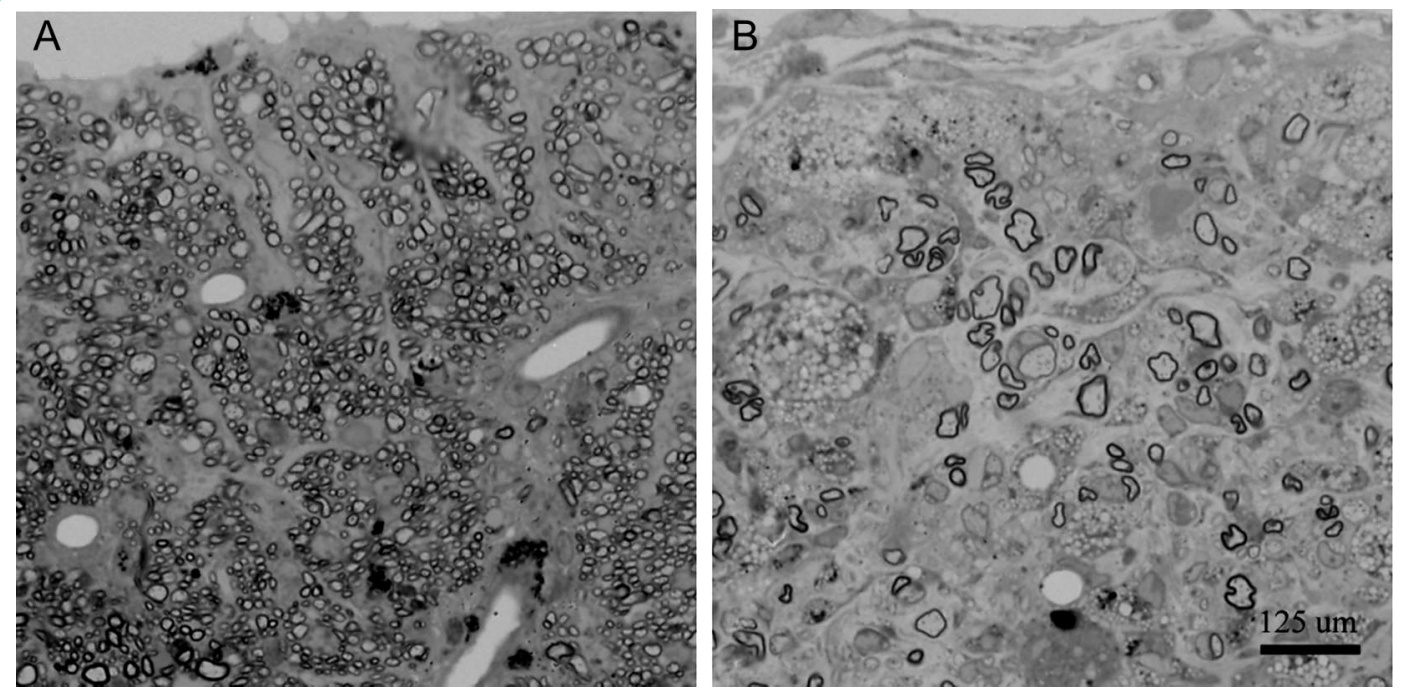

Light photomicrographs of spinal cord remyelination promoted by rHlgM22, a human immunoglobulin M (IgM) that binds to myelin and oligodendrocytes, in a virus-mediated model of chronic demyelination. An area of nearly complete remyelination is present in the ventral lateral spinal cord (A) of a mouse treated with a single dose of $50 \mu \mathrm{g}$ of rHlgM22 5 weeks earlier. Remyelination is characterized by densely packed thin myelin sheaths in relation to axon diameter. In contrast, a typical demyelinated lesion with minimal remyelination is observed in the spinal cord of a mouse treated with a control human IgM 5 weeks earlier (B), containing only a few remyelinated axons. Infiltrating macrophages in the demyelinating lesion are characterized by the presence of darkly stained debris-laden vesicles. A and $B$ are at the same magnification.

man mAbs bound to human oligodendrocytes. ${ }^{3}$ Using temporal lobe tissue from patients undergoing temporal lobectomy for epilepsy, we noted striking reactivity on the surface of human oligodendrocytes in vitro, ${ }^{3}$ which made us optimistic that these $\mathrm{mAbs}$ would be also effective at promoting remyelination in human patients (figure 2). We tested whether the mAb promotes remyelination by altering some aspect of the immune response, which was not the case. ${ }^{20}$ Instead, the $\mathrm{mAb}$ worked by activating oligodendrocytes. ${ }^{21}$

Development of a vector. We developed a vector capable of receiving any human heavy chain sequence and light chain sequence to generate large quantities of pure, recombinant $\mathrm{mAb} .{ }^{6}$ The vector incorporated methotrexate amplification system to eliminate most cells except those that produced large amounts of $\mathrm{mAb}$. We sequenced $\mathrm{sHIgM} 22^{22}$ and introduced the heavy and light chain into the vector. We then tested the recombinant protein ( $\mathrm{rHIgM} 22)$ in mice with demyelinating disease and demonstrated that the pure recombinant protein worked as well or better than the serum-derived protein. A subsequent dose titration experiment demonstrated that the dose necessary to induce remyelination was lower than our initial expectation. ${ }^{4}$ While our initial experiments used $0.5 \mathrm{mg}$ per mouse, we found that a single injection of 500 ng was all that was necessary to induce remyelination. Previous studies show that approximately $1 \%$ of the mAb enters the CNS. ${ }^{23}$ If a mouse weighing $20 \mathrm{~g}$ responds to a 500-ng dose, then the corresponding equivalent dose in a human patient weighing 50 or $70 \mathrm{~kg}$ would be approximately $2 \mathrm{mg}$.

CNS entry. Many individuals have thought that IgM mAbs would not enter the CNS. Our previous autoradiography studies showed that the mAbs did cross the blood-brain barrier. ${ }^{23}$ We performed a 7-Tesla MRI experiment in which we injected $m A b$ into animals infected with Theiler virus or experimental autoimmune encephalomyelitis. ${ }^{24}$ In both instances, CNS entry was demonstrated prominently by MRI. In contrast, in animals that had not been infected with virus or did not have demyelination, the $\mathrm{mAb}$ did not accumulate in the CNS. ${ }^{24}$ This indicated that $\operatorname{IgM} \mathrm{mAbs}$ can enter the demyelinating lesion and, specifically, bind to oligodendrocytes and myelin.

MRI as a surrogate marker of remyelination. Having previously demonstrated that MRI could serve as a surrogate marker for demyelination, for these experiments, we measured the total volume of lesions by voxels, and the total resulting pixels were calculated in three dimensions in both T1- and T2-weighted MRI scans. ${ }^{24}$ The animals chosen for this study had been infected with Theiler virus 6 to 7 months earlier and then given a single dose of human mAb. After 5 weeks, animals receiving saline showed slightly worsening MRI volumes. In contrast, the animals treated with the remyelination-promoting $\mathrm{mAb}$ rHIgM 22 showed a remarkable decrease in the lesion volume load in the spinal cord. ${ }^{24}$ Remarkably, these results 
Figure 2 rHIgM22 binds to the surface of oligodendrocytes
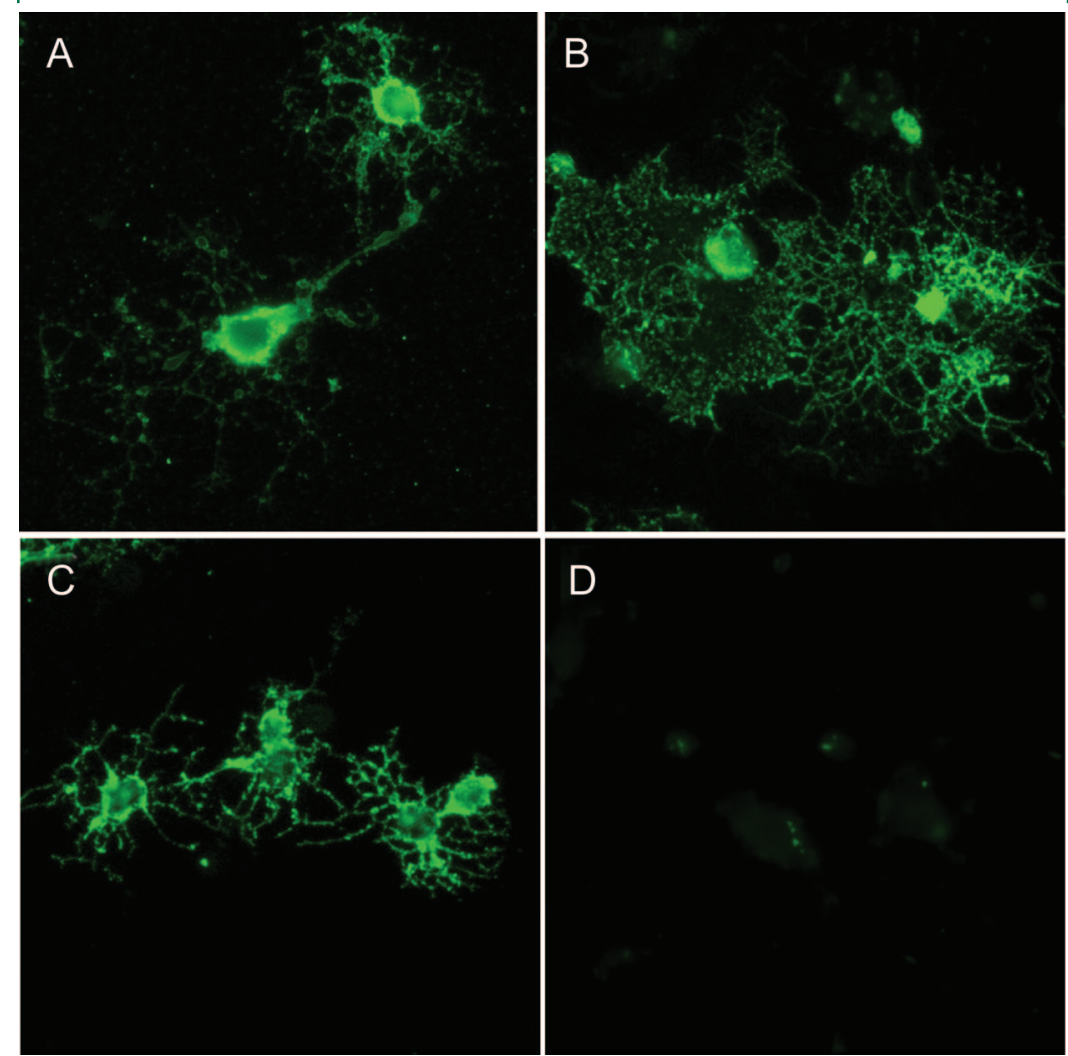

$\mathrm{D}$

The antigen recognized by $\mathrm{rH}$ lgM22 is conserved across a number of species. Immunoglobulin $\mathrm{M}$ binding was assessed via fluorescently conjugated secondary antihuman $\mu$-chainspecific monoclonal antibodies (mAbs). Panels depict surface immunolabeling of live cells with rHlgM22 on cultures of CNS glia derived from adult mouse (A), rat (B), and human (C) cerebral cortex. (D) Background staining with anti- $\mu$-chain secondary $m A$ bs alone.

were obtained after a single dose of the human $\mathrm{mAb}$, and almost every animal showed this response. We correlated areas of reduction of MRI signal with $1-\mu \mathrm{m}$-thick araldite-embedded sections of the spinal cord. There was a precise correlation between areas of reduction of MRI signal and areas of remyelination.

Testing for functional improvement. Using activity boxes to test for improvement in functional deficits by measuring both horizontal and vertical movements in treated mice, we observed mAb-induced improvements (figure 3). We compared animals treated with methylprednisolone to animals treated with methylprednisolone plus a single initial dose of rHIgM22 antibody. Methylprednisolone is the standard treatment in patients with acute attacks of multiple sclerosis (MS), and we wanted to ascertain that methylprednisolone would not interfere with mAb-induced remyelination. Day 12 following $\mathrm{mAb}$ administration demonstrated a marked improvement in the function of the mAb plus methylprednisolone-treated animals, compared to methylprednisolone-treated animals, which settled down to a higher baseline by 30 days (day of sacrifice and termination of the study).
AUTOANTIBODIES FOR THE ENHANCEMENT OF NEURITE OUTGROWTH In our quest to find a $\mathrm{mAb}$ that promotes remyelination, we had access to 125,000 samples of monoclonal proteins from patient donors with monoclonal gammopathies. Along with attaining our target goal of six sera human mAbs that bound to oligodendrocytes in the live cerebellum, we also inadvertently discovered $\mathrm{mAbs}$ that bind to the surface of neurons and enhance neuronal outgrowth, ${ }^{25}$ which we call sHIgM12 and sHIgM42. Both mAbs mediate neurite extensions (figure 4). The level of neurite extension is the same as when neurons are grown on a substrate of laminin. We then treated mice with a Theiler virus infection. Whereas the Theiler virus model is characterized by demyelination, the model is relevant to these studies of axon injury because the animals also develop extensive axonal degeneration and loss during the chronic phase of the disease. ${ }^{26}$ Animals had improved neurologic function beginning at 2 weeks following treatment, which persisted for 8 weeks, when we terminated the experiment (figure 5). Studies investigating whether there were histopathologic changes associated with improvement of function are underway.

Our laboratory has documented the binding properties of these $\mathrm{mAbs}$, and to date, we have seen successful binding to cerebellar neurons, retinal ganglion neurons, and cortical neurons. Therefore, these mAbs could have potentially important roles as therapeutic molecules in a number of disease processes in which axons and neurons die in the CNS, ${ }^{25}$ such as amyotrophic lateral sclerosis or stroke.

\section{NATURAL HUMAN AUTOANTIBODIES IN THE} TREATMENT OF MALIGNANCY Metastatic tumors to the brain constitute a major cause of death from malignancy. Our search for a remyelinating $\mathrm{mAb}$ also inadvertently led to the discovery of a $\mathrm{mAb}$ that binds to the dendritic cells of the immune system to induce cytotoxic $\mathrm{T}$ cells. The antigen appears to be a very important molecule in the treatment of malignancy (figure 6). ${ }^{5,27}$ Because the $\mathrm{mAb}$ works on peripheral dendritic cells and does not need to enter the CNS, it is useful for treating metastatic tumors in the CNS.

B7-DC-XAb and metastatic melanoma. This mAb binds to a molecule B7-DC on the surface of dendritic cells ${ }^{28}$ and is part of the co-stimulatory family of B7 proteins. Immature dendritic cells do not express detectable B7DC. However, when we treat dendritic cells with B7DC-XAb mAb, the B7-DC molecule becomes upregulated, thus activating the cells. ${ }^{29}$ The result is from binding of the $\mathrm{mAb}$ to the $\mathrm{B} 7$ molecule itself, since the $m A b$ fails to bind or stimulate dendritic cells from B7 knock-out mice. ${ }^{30}$ The mAb does not bind through the Fc receptor. As a result of dendritic cells 
Figure 3 A single dose of rHlgM22 combined with methylprednisolone twice a week alters spontaneous activity in mice with demyelinating disease

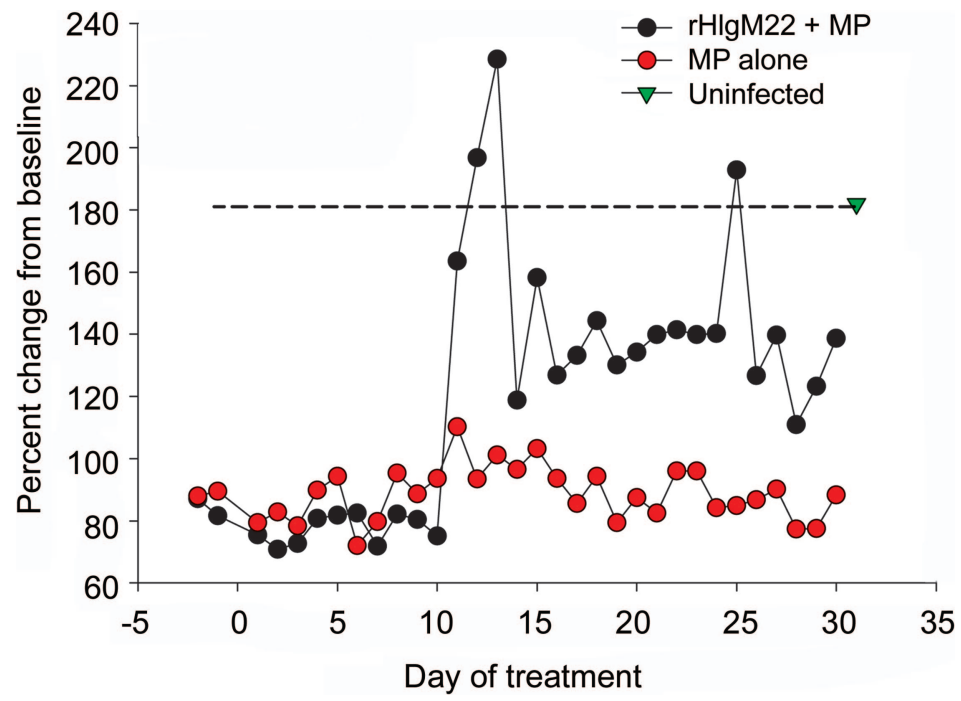

Methylprednisolone (MP) treatment (1 mg 2 times a week) was performed alone or combined with a single initial $500-\mu$ g bolus of rHlgM22. Groups of 5 mice 30 days after infection with TMEV were monitored continuously in activity boxes (Accuscan Inc.) which measure spontaneous horizontal and vertical movements by infrared beam breaks. Nocturnal activity, a time when mice are normally active, was used as a measure of a treatment group's ability and desire to explore their environment. By day 12 of treatment, spontaneous nocturnal horizontal activity of the MP + rHlgM22 group greatly increased and was comparable statistically to the uninfected control group. The nocturnal activity of virusinfected mice treated with MP alone did not change over the course of the study. From day 12 to 24 of treatment, the nocturnal activity of the MP + rHlgM22 group was comparable to the activity of a non-virus-infected group of mice. Statistical comparisons were by Mann-Whitney rank sum test of the values on each day of treatment.

becoming activated, they process tumor antigens to induce a vigorous cytotoxic T-cell antitumor response. ${ }^{5,28,30} \mathrm{We}$ asked if these mAbs could be useful in the treatment of malignancy.

Metastatic melanoma in an animal model. We chose metastatic melanoma as an experimental paradigm. We injected animals with B16 melanoma in a model of lung metastasis. The animals received $\mathrm{mAb}$ on days 3 through 5 following injection of the malignant cells. On day 25, animals were killed and examined for extent of lung metastasis. All mice treated with $30 \mu \mathrm{g}$ of B7-DC cross-linking antibody had dramatically reduced numbers of lung metastases, and several mice had complete resolution of the metastatic melanoma lesions. ${ }^{5}$

Therapeutic application of dendritic cell-activating mAbs. The most exciting aspect of this discovery is not in its potential to cure a single form of cancer but rather that it is an approach that can be applied to many types of malignancies. Dendritic cells circulate through tumors to process tumor antigens to be presented to $\mathrm{CD}^{+}{ }^{+} \mathrm{T}$ cells. ${ }^{31}$ In most malignant tumors, $\mathrm{CD}^{+}{ }^{+} \mathrm{T}$ cells are in a regulatory state and not a killing state. ${ }^{32}$ The cytokines (IL10, IL4, TGF- $\beta$ ) in malignancy render immune cells incapable of responding to the tumor
Figure 4 A human immunoglobulin M (IgM) that binds to neurons promotes neurite extension
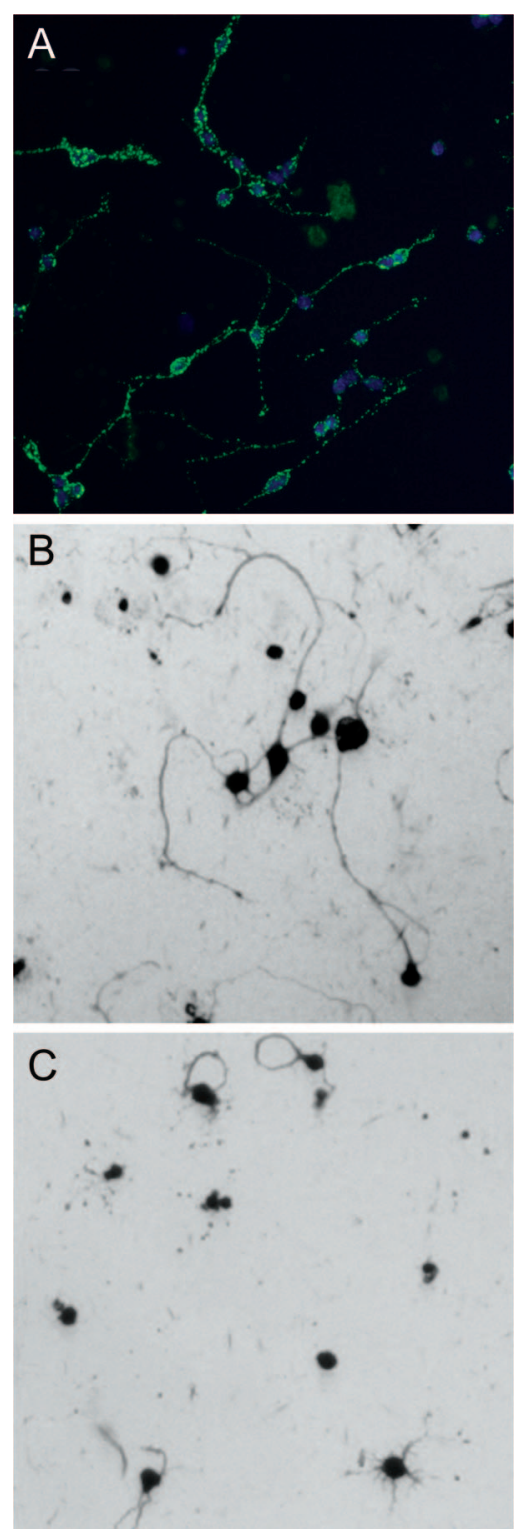

sHlgM42 is a serum-derived human monoclonal IgM that binds to the surface of many types of neurons. sHlgM42 binds to the surface of live rat cerebellar granule cell neurons (A). When rat granule cells are seeded onto surfaces coated with human sHlgM42, long neurite extension is induced within 24 hours (B). During the same time period, minimal neurites are extended by granule cells seeded onto an antioligodendrocyte monoclonal antibody (mAb)-coated surface (C) demonstrating the specificity of this natural human mAb. Cell architecture is revealed by Coomassie blue stain.

challenge. However, a single treatment with B7-DC$\mathrm{XAb}$ activates the dendritic cells to process more tumor antigens such that the peptides are now presented to the cytokine-secreting $\mathrm{CD}^{+} \mathrm{T}$ cells (interferon- $\gamma$ and interleukin-17), which then activate cytotoxic $\mathrm{CD}^{+} \mathrm{T}$ cells. This approach to tumor therapy may apply to a wide range of situations in which cytotoxic $T$ cells need to be activated to induce tumor regression. The $\mathrm{mAb}$ is 


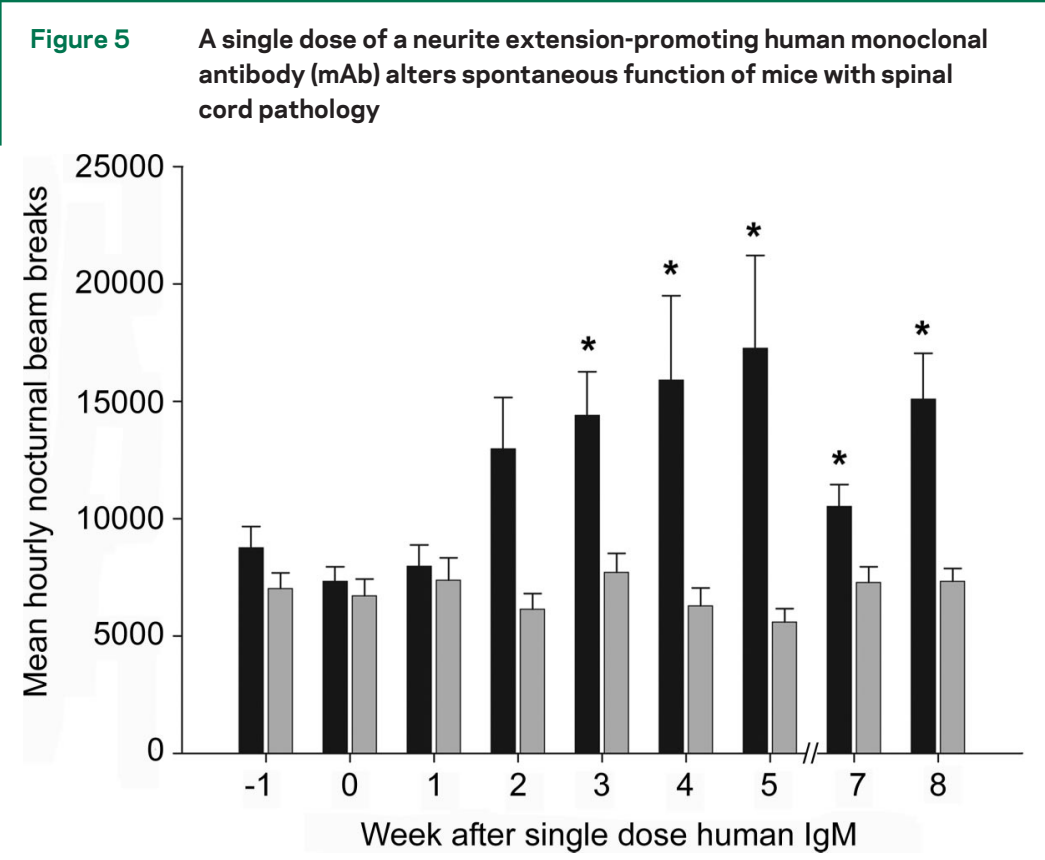

Mean hourly spontaneous nocturnal activity is altered within 3 weeks after a single intraperitoneal injection of $100 \mu \mathrm{g}$ human of sHlgM12. Groups of five mice with chronic TMEVinduced spinal cord demyelination and axon loss were treated with sHlgM12 (black bars) or a control human immunoglobulin M (IgM) that doses not bind to neurons (gray bars). Mice were monitored using AccuScan activity monitoring boxes for 72 consecutive hours per week. The mean \pm standard error of hourly horizontal activity over three 12-hour nocturnal periods (when mice are normally active) were compared to the nocturnal activity for the 3 nights before treatment (time 0 ). A difference in activity compared to pre treatment was present at 3 to 8 weeks after treatment ( ${ }^{*} p<0.01$ ) with sHlgM12, but not using a control human IgM mAb. Statistical comparisons of all time points by Kruskal-Wallis one way analysis of variance on ranks indicated a difference in activity. Specific time points with differences in nocturnal activity were then tested by one-way analysis of variance.

also effective in animal models of breast cancer and lymphoma.

THE LIPID RAFT The lipid raft is an integral component in the mechanism of action of therapeutic IgM mAbs. The mechanism of action of this class of mAbs appears to be very similar irrespective of whether the $\mathrm{mAb}$ is promoting remyelination, neurite outgrowth, or dendritic cell activation. The activation is through molecules on lipid rafts or lipid microdomains. All of these $\mathrm{mAbs}$ induce a transient increase in calcium influx in the recognized cells. ${ }^{21}$ The mAbs engage downstream signaling molecules that inform the cell to perform the primary functions of the cell. In the case of the oligodendrocyte, the primary function is myelin production. A primary function of the neuron is neurite extension and connectivity. A primary function of a dendritic cell is activation to process antigens to induce an immune response.

Lipid raft in the remyelination model. Noting that the mAbs cross-link antigens on the surface of oligodendrocytes, we observed that they cause molecules not normally in contact to come together and form a large molecular signaling complex. We performed calcium imaging experiments in which oligodendrocytes and astrocytes in culture were treated with remyelinating sHIgM22 mAb or control antibody. We observed two types of responses: a fast $\mathrm{Ca}^{++}$response mediated by astrocytes and a slower $\mathrm{Ca}^{++}$ response in oligodendrocytes. ${ }^{21}$ The delayed response is mediated via a calcium-dependent AMPA channel. Blocking the AMPA channel with CNQX stops calcium entry and blocks signaling within the cell. Signaling events taking place within the cell are blocked by this molecule. Additionally, the complete $\operatorname{IgM}$ molecule needs to be intact in order to activate the response. It appears that the cross-linking feature of the pentameric structure of $\operatorname{IgM}$ is necessary to activate oligodendrocytes by $\mathrm{rHIgM} 22$ or mAbO4. The more immediate $\mathrm{Ca}^{++}$response in astrocytes is mediated by phospholipase $\mathrm{C}$, another important signaling molecule. Again, fragmentation of the $\mathrm{mAb}$ to monomers or bivalent mAbs abrogates the response. Transient elevation of $\mathrm{Ca}^{++}$in cells is associated with cell survival, whereas persistent elevation of $\mathrm{Ca}^{++}$with cell death. ${ }^{33}$

Antibody-signaling response occurs by activating molecules at the level of lipid rafts, based on ultracentrifugation of plasma membrane preps. Lipid rafts are very important signaling microdomains on the surface of most cells. Disruption of the lipid microdomain by the detergent B-methylcyclodextrin prevents $\mathrm{Ca}^{++}$signaling. ${ }^{34}$ Exposure of oligodendrocytes to $\mathrm{rHIgM} 22$ or mouse $\mathrm{mAb} O 4$ at $15^{\circ} \mathrm{C}$ results in dramatic rearrangement of the lipid membrane binding to the mAbs observed by immunofluorescence.

Our concept is that pentameric IgM mAbs bind to molecules in lipid rafts, and this allows crosslinking of target molecules on the cell surface. The earliest event is the precise binding of the IgM molecule $\mathrm{mAb}$ to multiple unique antigens on the surface of the corresponding cell. This causes molecules that normally do not interact to come together to form a signaling complex. The final common pathway is a signal to the nucleus that activates the cell in question. ${ }^{28}$ One common pathway of how these mAbs work is by preventing apoptosis ${ }^{34}$ and activating $\mathrm{NF} \kappa \mathrm{B} \cdot{ }^{35}$

IMMINENT CLINICAL TRIALS B7-DC-XAb is already in a phase I clinical trial at the Mayo Clinic in patients with metastatic melanoma. In this situation, nonconcentrated hyperimmune serum from plasmapheresis of the patient naturally manufacturing this $\mathrm{mAb}$ is being transferred IV to patients with metastatic melanoma.

The first recombinant natural $\mathrm{mAb}$ to enter clinical trial will be rHIgM22. The mAb will be tested in patients with nonrecovering severe attacks of MS. 


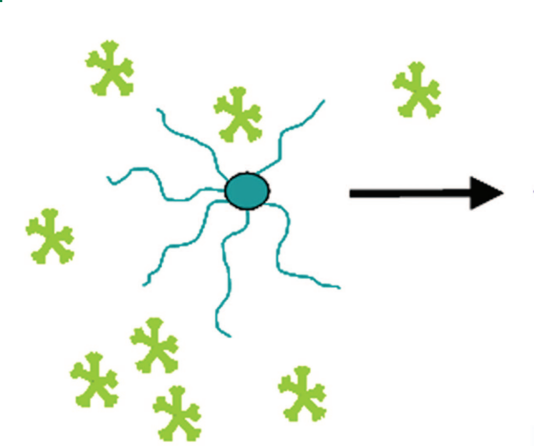

Immature dendritic cell

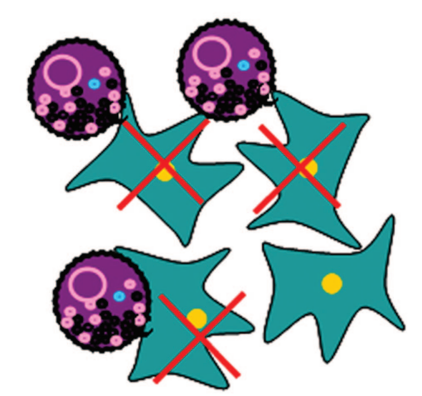

$\mathrm{CD}^{+} \mathrm{T}$ cells kill tumor cells

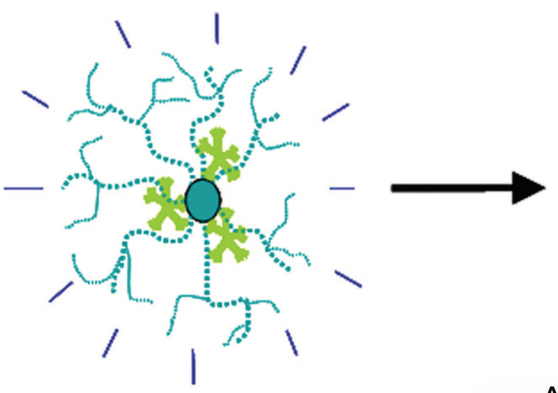

IgM binding induces intracellular signals

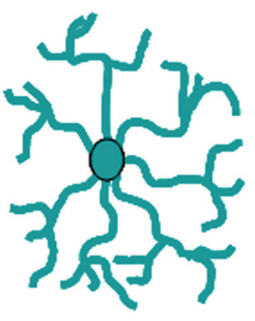

Activated dendritic cell

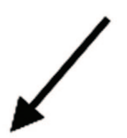

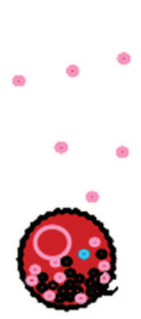

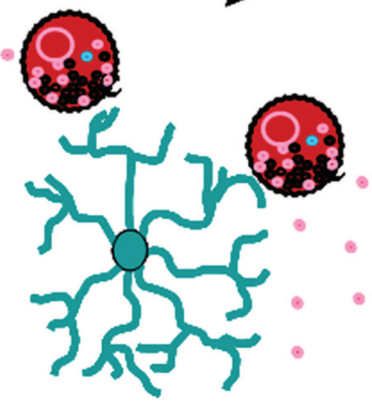

Dendritic cell recruits T cells which release IFN $\gamma$, IL17

B7-DC-XAb recognizes and binds to the surface of dendritic cells. The pentameric immunoglobulin M (green) mAb clusters portions of the cell membrane, which leads to calcium influx and dendritic cell activation. The activated dendritic cells interact with $\mathrm{CD}^{+}{ }^{+} \mathrm{T}$ cells, which secrete cytokines to alter the systemic immune response. This mAb-induced change in immune function is sufficient to stimulate cytotoxic $\mathrm{CD} 8^{+} \mathrm{T}$ cells to clear malignant metastatic B16 tumor cells in vivo.

This would be the first attempt to enhance remyelination in an MS lesion with a completely different therapeutic approach to the present MS treatments. In this case, the therapy would target the cells that make the myelin sheath, in contrast to all other Food and Drug Administration-approved MS treatments, which target immune cells.

However, a successful clinical trial of rHIgM22 to promote remyelination would have an impact much larger than a specific benefit to patients with MS. The use of natural autoantibodies is potentially an important therapeutic technique in combating a wide spectrum of neurologic and malignant disease. Monoclonal antibodies or mAbs that bind to the surface of neurons to enhance neuronal outgrowth and prevent axon death could provide a new approach to treatment of traumatic spinal cord injury, amyotrophic lateral sclerosis, primary motor peripheral neuropathies, or even stroke. Similarly, we could potentially have a new, powerful weapon in our cancer-fighting arsenal as we discover those mAbs that activate dendritic cells to generate cytotoxic responses to specific types of tumors. The effect of these mAbs on various neurologic disorders will be tested in phase II trials, assuming they are found safe in phase I trials.

\section{ACKNOWLEDGMENT}

The authors thank Lea Dacy for initial preparation of the manuscript from a transcript of the Frontiers in Neuroscience lecture at the American Academy of Neurology delivered on April 17, 2008, by Dr. Moses Rodriguez.

Received October 14, 2008. Accepted in final form January 23, 2009.

\section{REFERENCES}

1. Coutinho A, Kazatchkine MD, Avrameas S. Natural autoantibodies. Curr Opin Immunol 1995;7:812-818.

2. Avrameas S, Ternynck T, Tsonis IA, Lymberi P. Naturally occurring B-cell autoreactivity: a critical overview. J Autoimmun 2007;29:213-218.

3. Warrington AE, Asakura K, Bieber AJ, et al. Human monoclonal antibodies reactive to oligodendrocytes promote remyelination in a model of multiple sclerosis. Proc Natl Acad Sci USA 2000;97:6820-6825.

4. Warrington AE, Bieber AJ, Ciric B, Pease LR, Van Keulen $\mathrm{V}$, Rodriguez M. A recombinant human IgM promotes myelin repair after a single, very low dose. J Neurosci Res 2007;85:967-976.

5. Radhakrishnan S, Nguyen LT, Ciric B, et al. Immunotherapeutic potential of B7-DC (PD-L2) cross-linking anti- 
body in conferring antitumor immunity. Cancer Res 2004;64:4965-4972.

6. Mitsunaga Y, Ciric B, Van Keulen V, et al. Direct evidence that a human antibody derived from patient serum can promote myelin repair in a mouse model of chronicprogressive demyelinating disease. Faseb J 2002;16:13251327.

7. Bieber AJ, Warrington A, Pease LR, Rodriguez M. Humoral autoimmunity as a mediator of CNS repair. Trends Neurosci 2001;24:S39-44.

8. Asakura K, Miller DJ, Pease LR, Rodriguez M. Targeting of IgM kappa antibodies to oligodendrocytes promotes CNS remyelination. J Neurosci 1998;18:7700-7708.

9. Lang W, Rodriguez M, Lennon VA, Lampert PW. Demyelination and remyelination in murine viral encephalomyelitis. Ann NY Acad Sci 1984;436:98-102.

10. Rodriguez M, Lennon VA, Benveniste EN, Merrill JE. Remyelination by oligodendrocytes stimulated by antiserum to spinal cord. J Neuropathol Exp Neurol 1987;46:84-95.

11. Rodriguez M, Lennon VA. Immunoglobulins promote remyelination in the central nervous system. Ann Neurol 1990;27:12-17.

12. Rodriguez M, Pierce ML, Thiemann RL. Immunoglobulins stimulate central nervous system remyelination: electron microscopic and morphometric analysis of proliferating cells. Lab Invest 1991;64:358-370.

13. Miller DJ, Sanborn KS, Katzmann JA, Rodriguez M. Monoclonal autoantibodies promote central nervous system repair in an animal model of multiple sclerosis. J Neurosci 1994;14:6230-6238.

14. Miller DJ, Bright JJ, Sriram S, Rodriguez M. Successful treatment of established relapsing experimental autoimmune encephalomyelitis in mice with a monoclonal natural autoantibody. J Neuroimmunol 1997;75:204-209.

15. Miller DJ, Njenga MK, Parisi JE, Rodriguez M. Multiorgan reactivity of a monoclonal natural autoantibody that promotes remyelination in a mouse model of multiple sclerosis. J Histochem Cytochem 1996;44:1005-1011.

16. Asakura K, Miller DJ, Murray K, Bansal R, Pfeiffer SE, Rodriguez M. Monoclonal autoantibody SCH94.03, which promotes central nervous system remyelination, recognizes an antigen on the surface of oligodendrocytes. J Neurosci Res 1996;43:273-281.

17. Asakura K, Miller DJ, Pogulis RJ, Pease LR, Rodriguez M. Oligodendrocyte-reactive O1, O4, and HNK-1 monoclonal antibodies are encoded by germ line immunoglobulin genes Brain Res Mol Brain Res 1995;34:283-293.

18. Miller DJ, Rodriguez M. A monoclonal autoantibody that promotes central nervous system remyelination in a model of multiple sclerosis is a natural autoantibody encoded by germ line immunoglobulin genes. J Immunol 1995;154: 2460-2469.

19. Bieber AJ, Warrington A, Asakura K, et al. Human antibodies accelerate the rate of remyelination following lysolecithin-induced demyelination in mice. Glia 2002;37: 241-249.

20. Ciric B, Van Keulen V, Paz Soldan M, Rodriguez M, Pease LR. Antibody-mediated remyelination operates through mechanism independent of immunomodulation. J Neuroimmunol 2004;146:153-161.

21. Paz Soldan MM, Warrington AE, Bieber AJ, et al Remyelination-promoting antibodies activate distinct $\mathrm{Ca} 2+$ influx pathways in astrocytes and oligodendrocytes: relationship to the mechanism of myelin repair. Mol Cell Neurosci 2003;22:14-24.

22. Ciric B, VanKeulen V, Rodriguez M, Kyle RA, Gertz MA, Pease LR. Clonal evolution in Waldenstrom macroglobulinemia highlights functional role of B-cell receptor. Blood 2001;97:321-323.

23. Hunter SF, Miller DJ, Rodriguez M. Monoclonal remyelination-promoting natural autoantibody $\mathrm{SCH}$ 94.03: pharmacokinetics and in vivo targets within demyelinated spinal cord in a mouse model of multiple sclerosis. J Neurol Sci 1997;150:103-113.

24. Pirko I, Ciric B, Gamez J, et al. A human antibody that promotes remyelination enters the CNS and decreases lesion load as detected by T2-weighted spinal cord MRI in a virus-induced murine model of MS. Faseb J 2004;18: 1577-1579.

25. Warrington AE, Bieber AJ, Van Keulen V, Ciric B, Pease LR, Rodriguez M. Neuron-binding human monoclonal antibodies support central nervous system neurite extension. J Neuropathol Exp Neurol 2004;63:461-473.

26. McGavern DB, Murray PD, Rivera-Quinones C, Schmelzer JD, Low PA, Rodriguez M. Axonal loss results in spinal cord atrophy, electrophysiological abnormalities and neurological deficits following demyelination in a chronic inflammatory model of multiple sclerosis. Brain 2000;123 Pt 3:519-531.

27. Van Keulen VP, Ciric B, Radhakrishnan S, et al. Immunomodulation using the recombinant monoclonal human B7-DC cross-linking antibody rHIgM12. Clin Exp Immunol 2006;143:314-321.

28. Blocki FA, Radhakrishnan S, Van Keulen VP, et al. Induction of a gene expression program in dendritic cells with a cross-linking IgM antibody to the co-stimulatory molecule B7-DC. Faseb J 2006;20:2408-2410.

29. Pavelko KD, Heckman KL, Schenk EL, Radhakrishnan S, Pease LR. Differential tumor-specific immunity using the immune modulator B7-DC Xab and live tumor or tumor lysate vaccines. J Immunother 2007;30:900.

30. Nguyen LT, Radhakrishnan S, Ciric B, et al. Cross-linking the B7 family molecule B7-DC directly activates immune functions of dendritic cells. J Exp Med 2002;196:13931398.

31. Heckman KL, Schenk EL, Radhakrishnan S, Pavelko KD, Hansen MJ, Pease LR. Fast-tracked CTL: rapid induction of potent anti-tumor killer T cells in situ. Eur J Immunol 2007;37:1827-1835.

32. Radhakrishnan S, Nguyen LT, Ciric B, Van Keulen VP, Pease LR. B7-DC/PD-L2 cross-linking induces NF-kappa $\mathrm{B}$-dependent protection of dendritic cells from cell death. J Immunol 2007;178:1426-1432.

33. Rimessi A, Giorgi C, Pinton P, Rizzuto R. The versatility of mitochondrial calcium signals: from stimulation of cell metabolism to induction of cell death. Biochim Biophys Acta 2008;1777:808-816.

34. Howe CL, Bieber AJ, Warrington AE, Pease LR, Rodriguez M. Antiapoptotic signaling by a remyelinationpromoting human antimyelin antibody. Neurobiol Dis 2004;15:120-131.

35. Radhakrishnan S, Wiehagen KR, Pulko V, et al. Induction of a Th1 response from Th2-polarized $\mathrm{T}$ cells by activated dendritic cells: dependence on TCR:peptide-MHC interaction, ICAM-1, IL-12, and IFN-gamma. J Immunol 2007;178:3583-3592. 